\title{
Interaction Studies
}

\section{Robot-Mediated interviews: A field trial with a potential real world user --Manuscript Draft--}

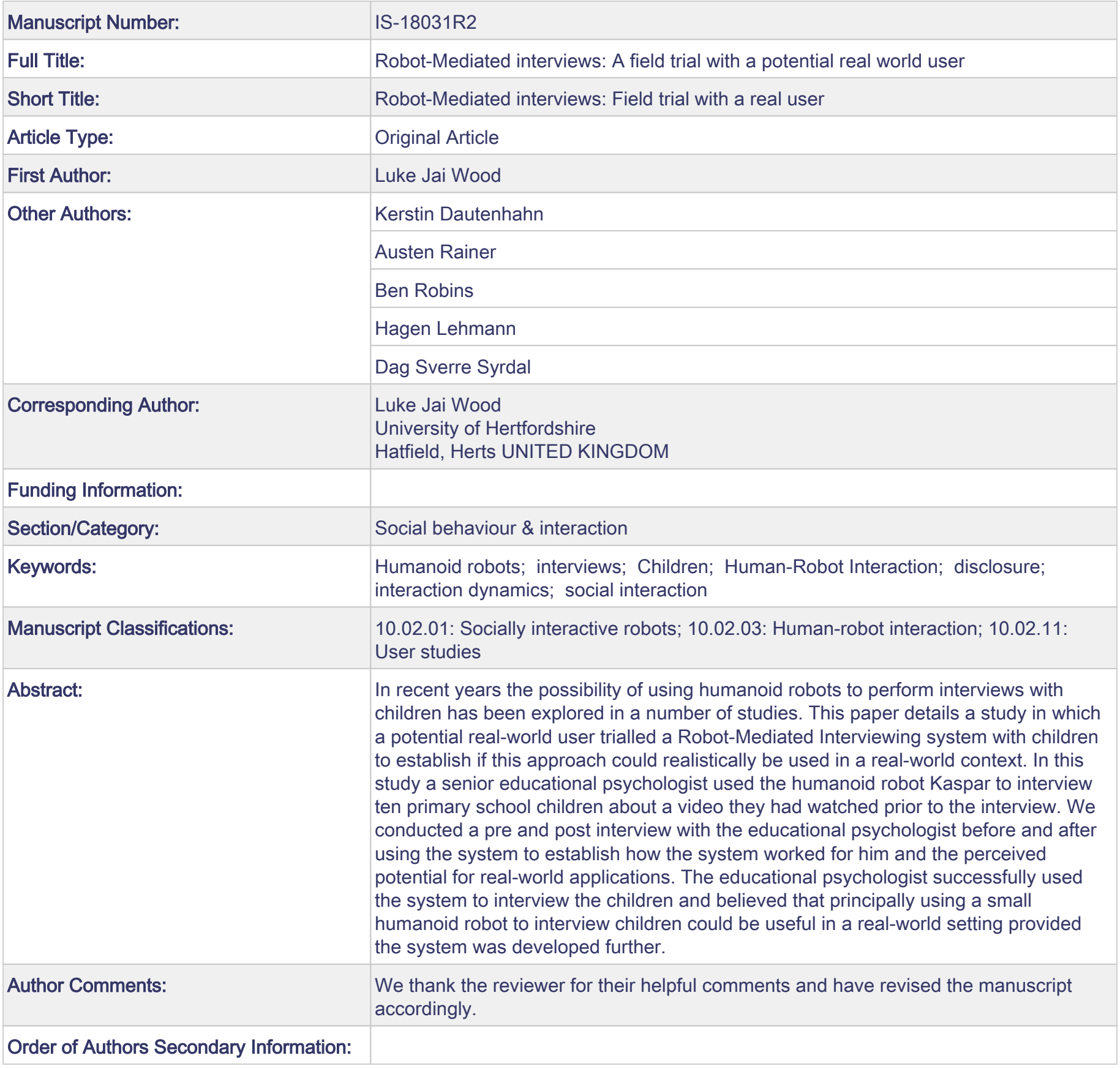




\section{Biographical note}

Luke Wood is a Research Fellow in the Adaptive Systems Research Group at the University of Hertfordshire, UK. He completed his PhD in 2015, the title of his thesis is "Robot-mediated interviews: a robotic intermediary for facilitating communication with children". Luke currently works for the Horizon2020 project Babyrobot which studies robot-assisted therapy for children with autism. Luke's research is focusing on assistive technology for facilitating communication and interaction for children with special needs. His research includes hardware and software development as well as designing, running and analysing human-robot interaction studies. 
${ }^{1}$ Luke Jai Wood: 1.wood@herts.ac.uk

${ }^{2}$ Kerstin Dautenhahn: k.dautenhahn@herts.ac.uk

Austen Rainer: a.w.rainer@gmail.com

Ben Robins: b.robins@herts.ac.uk

Hagen Lehmann: hagen.lehmann@gmail.com

Dag Sverre Syrdal: dagsyrdal@gmail.com

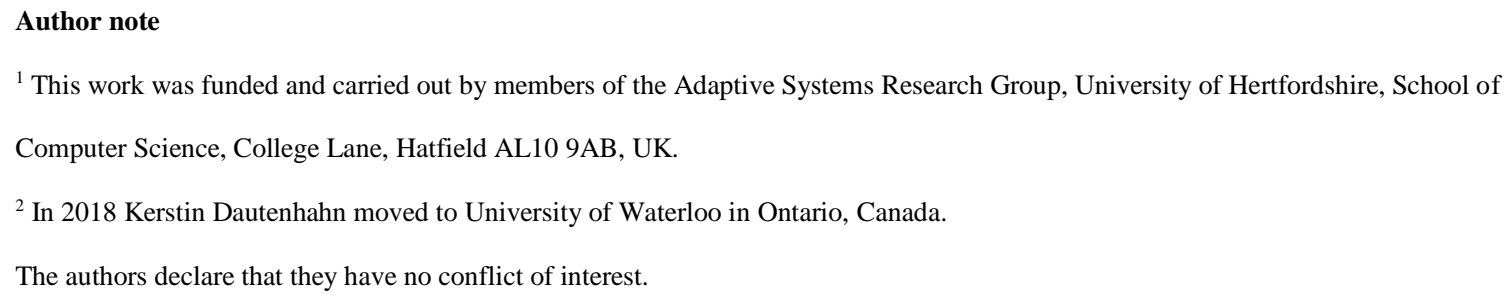




\begin{abstract}
In recent years the possibility of using humanoid robots to perform interviews with children has been explored in a number of studies. This paper details a study in which a potential real-world user trialled a Robot-Mediated Interviewing system with children to establish if this approach could realistically be used in a real-world context. In this study a senior educational psychologist used the humanoid robot Kaspar to interview ten primary school children about a video they had watched prior to the interview. We conducted a pre and post interview with the educational psychologist before and after using the system to establish how the system worked for him and the perceived potential for real-world applications. The educational psychologist successfully used the system to interview the children and believed that principally using a small humanoid robot to interview children could be useful in a real-world setting provided the system was developed further.
\end{abstract}

Keywords: 


\section{Introduction}

Previous studies in the area of Robot-Mediated Interviews have demonstrated that children respond to a robotic interviewer in a similar manner to which they do a human interviewer regardless of question difficulty (Wood, Dautenhahn, et al., 2013a, 2013b; Wood, Dautenhahn, Rainer, et al., 2013). Further to this, feedback from potential real-world users indicated that a Robot-Mediated approach to interviewing children might be useful in a number of areas provided that the system was sufficiently flexible (Wood et al., 2014). The study presented in this paper takes the next logical step, trialling the humanoid robot Kaspar as a Robot-Mediated Interviewing system with a potential real-world user. The Kaspar robot is a small child sized humanoid robot that has a realistic human face and is capable of performing different gestures and facial expressions to facilitate interactions (Wood et al., 2019). If robots such as Kaspar are to be used to conduct interviews in realworld settings, it is important that the interfaces are designed and tested with real-world users.

The study outlined in this paper involved a pre-trial introduction and interview with the expert trialling the system to acquire a detailed description of their professional background and experience, along with their expectations of the system being trialled. During this introduction the expert was also given an overview of the arrangements for the study. The pre-trial was followed by a study in a school where the expert interviewed 10 children in a mainstream school individually regarding a 3 minute video they had watched prior to the interview via the Kaspar robot. The study then concluded with a post-trial interview with the expert to gather feedback on the system. The primary purpose of this 
study was a usability proof-of-concept test to establish if humanoid robots such as Kaspar could effectively be used in an interview setting by an expert working with children.

Further to this we also wanted to gather additional data to ascertain if experts working with children think that a Robot-Mediated Interview approach could be useful in their field, and if so, what experts working with children would require from a Robot-Mediated Interviewing system. Trialling the system with a potential real-world user is a critical step in establishing if this approach could be feasible outside of a research context and ultimately have real-world implications.

The three previous studies that we carried out as part of our research programme have shown that both neurotypical children and children with special needs respond well to the Kaspar robot in an interview setting (Wood, Dautenhahn, et al., 2013a, 2013b; Wood, Dautenhahn, Rainer, et al., 2013). Results gained from focus groups with potential expert users led to modifications being implemented to make the system more flexible, as suggested by the user panels. However, we needed to test the system with a real-world user in order to further highlight any unforeseen issues.

This paper contributes to the knowledge of the scientific community by taking the use of Robot-Mediated Interviews a step further and putting the system in the hands of a potential real-world user, gathering feedback about the usability of the system and its most beneficial applications. It is essential to gather real-world opinions from professionals who would potentially want to use such a system in order to establish the practical applications 
and what would be required from the system in terms of usability to make the transition from research to a real-world system. 


\section{Background}

Interviewing children can be a challenging task particularly when conducing an interview with a child that has special needs (Mencap, 1999); interviews are therefore carried out by highly trained experts. When a child is involved in a formal interview very often emotionally provocative questions have to be asked, which can be quite stressful for the child. Although police officers who conduct interviews with young children undergo extensive training it can be difficult for the interviewer to maintain their composure when the child is describing a stressful or traumatic ordeal. In such cases interviewers may subtly and unintentionally indicate their thoughts and feelings to the child through body language or facial expressions, which can have a detrimental effect on the child's disclosure (UK Government, 2011). Moreover the mere perception of a person's authority can sometimes have an effect on a witness, particularly with regards to suggestibility. Using a robot to conduct interviews with children could negate these problems because the robot's expressions and behaviours can be precisely controlled and it is clearly not an adult and as such may not be viewed in the same way.

In recent years the use of robots for social and therapeutic purposes has become a very popular area of research (Cabibihan, Javed, Ang Jr, \& Aljunied, 2013; Conti, Di Nuovo, Buono, \& Di Nuovo, 2017; Costa, Lehmann, Dautenhahn, Robins, \& Soares, 2015; Costescu, Vanderborght, \& David, 2014; Martínez-Miranda, Pérez-Espinosa, EspinosaCuriel, Avila-George, \& Rodríguez-Jacobo, 2018; Mead et al., 2018; Meghdari et al., 2016). Robots that assist children who have autism with social mediation and communication in particular show some positive results and potential (Chevalier, Martin, 
Isableu, Bazile, \& Tapus, 2017; Esteban et al., 2017; Pennisi et al., 2015; Robins \& Dautenhahn, 2014; Robins, Dautenhahn, \& Dickerson, 2009; Sartorato, Przybylowski, \& Sarko, 2017). Moving towards the emerging area of Robot-Mediated Interviews, Bethel et al. (C. L. Bethel, Stevenson, \& Scassellati, 2011) investigated whether children are as likely to share a secret with a humanoid robot as they are with an adult. Bethel et al. revealed that the children would readily interact with the robots and speak to them in a similar manner as they would an adult.

Our first study involved twenty-one children aged between 7 and 9 taking part in two interviews, one with an adult and one with a humanoid robot. The questions in the interviews focused on a special event that had recently taken place in the school. The results revealed that the children interacted with the robot in a very similar manner to how they interacted with a human interviewer (Wood, Dautenhahn, Rainer, et al., 2013).

Building on this study we investigated how twenty children aged between 7 and 9 would respond to questions of varying difficulty from a robotic interviewer compared to a human interviewer. The procedure was identical to the first study except that the main questions in the interviews focused on the theme of pets and animals. After each interview the children were asked to rate the difficulty of the questions and particular aspects of the experience. The results were consistent with our first study and previous work, providing both interviewers with similar information and amounts of information regardless of question difficulty (Wood, Dautenhahn, et al., 2013b). 
When examining the literature it became apparent that children with a disability are much more likely to be a victim of abuse than typically developing children (Jones et al., 2012). Despite this the number of prosecutions is relatively low with one of the factors influencing this being communication difficulties. Because robots have been used so successfully with children that have special needs previously (Cabibihan et al., 2013; Robins \& Dautenhahn, 2014; Robins et al., 2009), we conducted a small study with five children aged between 9 and 11 to investigate how children with special needs would respond to a robot in an interview setting. The results from quantitative data analysis revealed that the children interacted with Kaspar similarly to how they interacted with the human interviewer, providing both interviewers with similar information and amounts of information regardless of question difficulty. However qualitative analysis suggested that some children may have been more engaged with the robotic interviewer (Wood, Dautenhahn, et al., 2013a).

Exploring another important aspect of Robot-Mediated Interviews, Bethel et al. investigated if an eyewitness testimony would be influenced by a robotic interviewer compared to a human interviewer when presented with post event misinformation (Cindy $\mathrm{L}$ Bethel, Eakin, Anreddy, Stuart, \& Carruth, 2013). The study consisting of 101 adult participants viewed a slideshow of a crime being committed and afterwards were then asked some questions about the slide show by either a NAO robot or a human interviewer. In the misinformation condition the participants would be asked questions with misinformation embedded within the questions, whereas in the control condition no misinformation was embedded. The results of this study found that the participants were 
likely to be misled by the human interviewer, but not the robotic interviewer. Although this study was not conducted with children, the results appear to be encouraging for the potential of Robot-Mediated Interviews, as the study would seem to suggest that a robot could acquire more accurate information from the interviewee, which would be advantageous in a legal setting. Subsequent studies have also indicated that children with ASD were less likely to be misled by an avatar than a human interviewer (Hsu \& Teoh, 2017).

Evaluating the background of technology and describing how it has impacted our legal systems, criminal investigations and in particular, children's forensic testimonies, Kyriakidou reviews many of the studies conducted to date on Robot-Mediated Interviews (Kyriakidou, 2016). Kyriakidou highlights the ethical implications of using robots to interview children, and suggests research methodological designs that may benefit studies exploring robot interviews with children such as a robot exposure phase prior to the main data collection. Although only mentioned briefly in previous papers, our earlier studies with children all contained a familiarisation phase prior to the data collection phase (Wood, Dautenhahn, et al., 2013a, 2013b; Wood, Dautenhahn, Rainer, et al., 2013). However, as Kyriakidou suggests, further consideration needs to be given to question of how much the children are told about the autonomy of the robot and how it is controlled. In all of our previous studies the children were given the opportunity to control the robot and a full explanation of how the robot worked after the data gathering phase. If robots are to be used in a real-world setting the ethical implications of this will need further consideration, 
particularly when what the children are telling the robot could have such large implications on their lives.

A recent study investigated if children would be more likely to disclose occurrences of bullying to a robot or a human (C. L. Bethel et al., 2016). The sixty children participating in the study were between the ages of 8 and 12 and were split evenly between human and robotic interviewer conditions. The results from this study revealed that participants were significantly more likely to report that fellow students were teased about their looks to the robot interviewer in comparison to the human interviewer, which provides further encouraging evidence for the potential of Robot-Mediated Interviewers in the future.

Because many field trials indicated that Robot-Mediated Interviews may have some potential we began to investigate what the experts (i.e. specialist child interviewers) would require from such a system for it to work in a real-world setting. To determine the needs of such expert users we conducted three user panels with groups of potential real-world users to gather their views on our current system based on previous studies (Wood, Dautenhahn, et al., 2013a, 2013b; Wood, Dautenhahn, Rainer, et al., 2013) and find out what they would require for the system to be useful to them (Wood et al., 2014, 2016). The user groups consisted of specialist police officers, intermediaries, educational specialists and healthcare specialists. All three panels shared the view that for a Robot-Mediated Interviewing system to be useful in the real-world, it would need to be sufficiently flexible to follow any line of questioning. The most common recommendation by the participants in all three panels was to implement a direct speech interface that converts the voice of the 
interviewer allowing the interviewer to respond to any unexpected and spontaneous paths that an interview may take, rather than having scripted responses to choose from. In addition to this, the panels emphasised the importance of the interviewing being able to focus on interviewing the child rather than controlling the robot and as such the system may need some automated features to facilitate this. The feedback from this study equipped us with a specification for a feature set to implement before conducting the study that is the focus of this paper.

\section{Research questions}

The research questions this study aimed to answer were as follows:

RQ 1) What would experts working with children require from a Robot-Mediated Interviewing system?

Experts working with children quite often are not roboticists or computer scientists. For a Robot-Mediated Interviewing system to be useful in the real-world, the system would need to be sufficiently flexible and user friendly to be useable by non-technical staff. Therefore, gathering the relevant information as to what realworld users would require from such a system is essential for producing a useful system with real-world potential.

RQ 2) Can robots such as Kaspar be used in an interview setting by an expert working with children?

To ultimately establish if robots could be used as intermediaries for facilitating communication with children in an interview setting, the system needs be to put in the hands of an expert to gather feedback on how the system performs and whether 
the expert considers that a Robot-Mediated Interviewing approach would be useful in a real-world situation. 


\section{Methodology}

\section{Technical prerequisites implemented in the system}

In previous studies that we conducted, the questions and interview structure was highly scripted and focused on a single topic (Wood, Dautenhahn, et al., 2013a, 2013b; Wood, Dautenhahn, Rainer, et al., 2013). In a real-world setting an interview with a child is unpredictable and as such the system would need to be much more flexible. Prior to commencing this study, the feedback obtained from user panels (Wood et al., 2014, 2016) we previously conducted was considered and the most important elements were implemented, these being:

- A direct microphone link to the robot with voice conversion to increase the flexibility of the system.

- Automated behaviours to allow the interviewer to focus on the child rather than controlling the robot.

- A selection of gestures that could be activated by the onscreen Graphical User Interface (GUI).

Voice conversation system - In the previous studies that we conducted the robot was preprogramed with questions to ask the children and the robot was unable to go outside of the constraints of the main topic. In a real-world setting this is far too restrictive and would need to be much more flexible. To resolve this issue a direct microphone link with a piece of voice conversion was implemented. The software used to implement the voice conversion was (Screaming Bee) MorphVOX Pro 4.4.8. The software allowed for detailed 
customisation of the voice projected, which suited our needs. For the purpose of our study we required the voice of the interviewer to sound childlike, robotic, but most importantly, understandable. In previous studies some of the children had difficulty understanding the robot's voice, which had been generated from text to speech software. Taking this into consideration, the customisation of the voice output was very important and needed to be constructed carefully. To strike the correct balance many different settings were tried and tested in the software. When testing the different settings, a non-native English speaker was present to give his feedback on the clarity of the voice. This was important, because understanding a voice with an accent is often more difficult for non-native speakers, therefore making him an ideal candidate to test the intelligibility of the voice. The settings that were finally chosen increased the pitch of the voice slightly to make it sound more childlike, and a slight de-tune effect was implemented to create a more robotic tone and reinforce the impression of the robot. To thoroughly test the clarity of the voice, the principal investigator spoke to the tester in a remote location with the voice conversion activated and asked him to repeat what was being said. By testing the voice in this manner it was possible to provide an accurate indication of how easy the voice was to understand.

Automated behaviours - In our previous studies the voice and sequence of movements were scripted and were activated by keypress. Because the flexibility of the system was being increased with the implementation of direct voice conversion, some other aspects of the robot's other behaviour needed further consideration. During our user panel studies it was stated that the system should allow the interviewer to focus on interviewing the child, rather than operating the system. In order to do this the blinking and mouth movements of 
the robot were automated. The blinking of the robot was generated randomly within a set threshold by a timer. In addition to this, automated mouth movements for the robot were used to maintain the impression that the robot was talking whilst the interviewer was asking questions via the robot. The code used to achieve the mouth movement implemented a simple threshold mechanism. The openness of the robot's mouth would increase according to how loud the interviewer spoke; if the interviewer was not speaking the mouth would be closed. It is important to note that we were not looking to create lipsync.

Gestures implemented - A selection of gestures were implemented in the software, in accordance with the feedback gathered from the previously conducted user panels. The gestures were activated via a GUI, which can be seen in Figure 1. A selection of the robot's gestures can be seen in Figure 2.

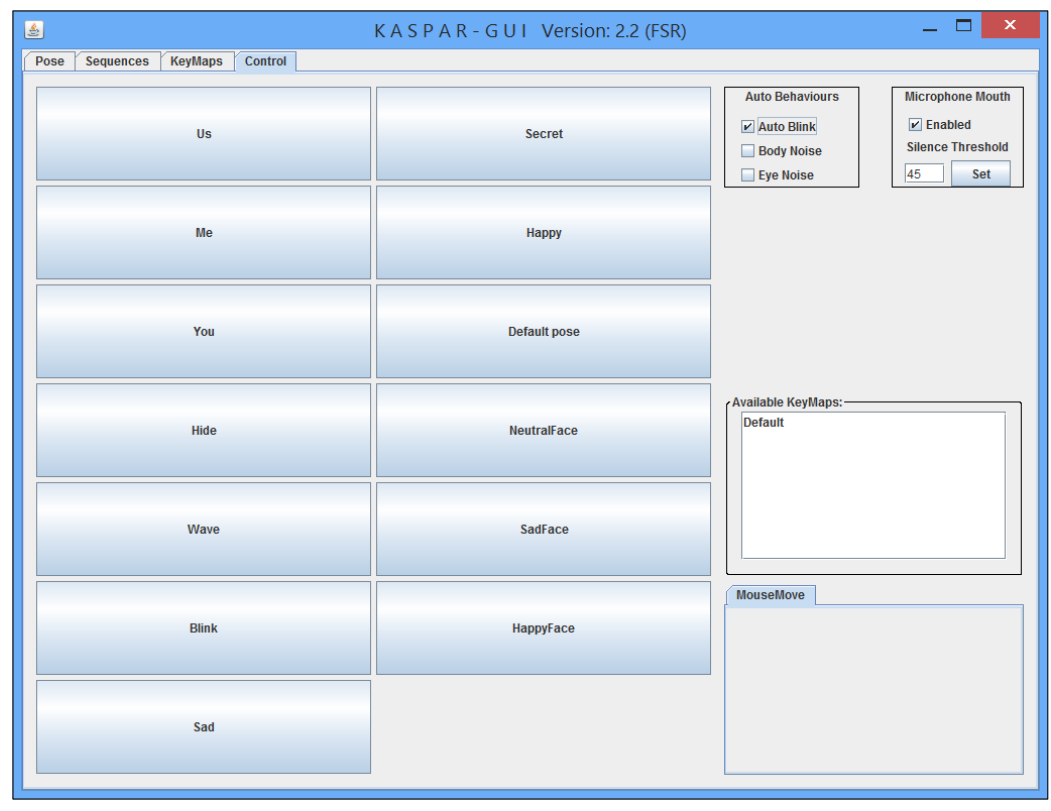


Figure 1. Screenshot of modified Kaspar Graphical User Interface (GUI). Note: additional features in top right hand corner.

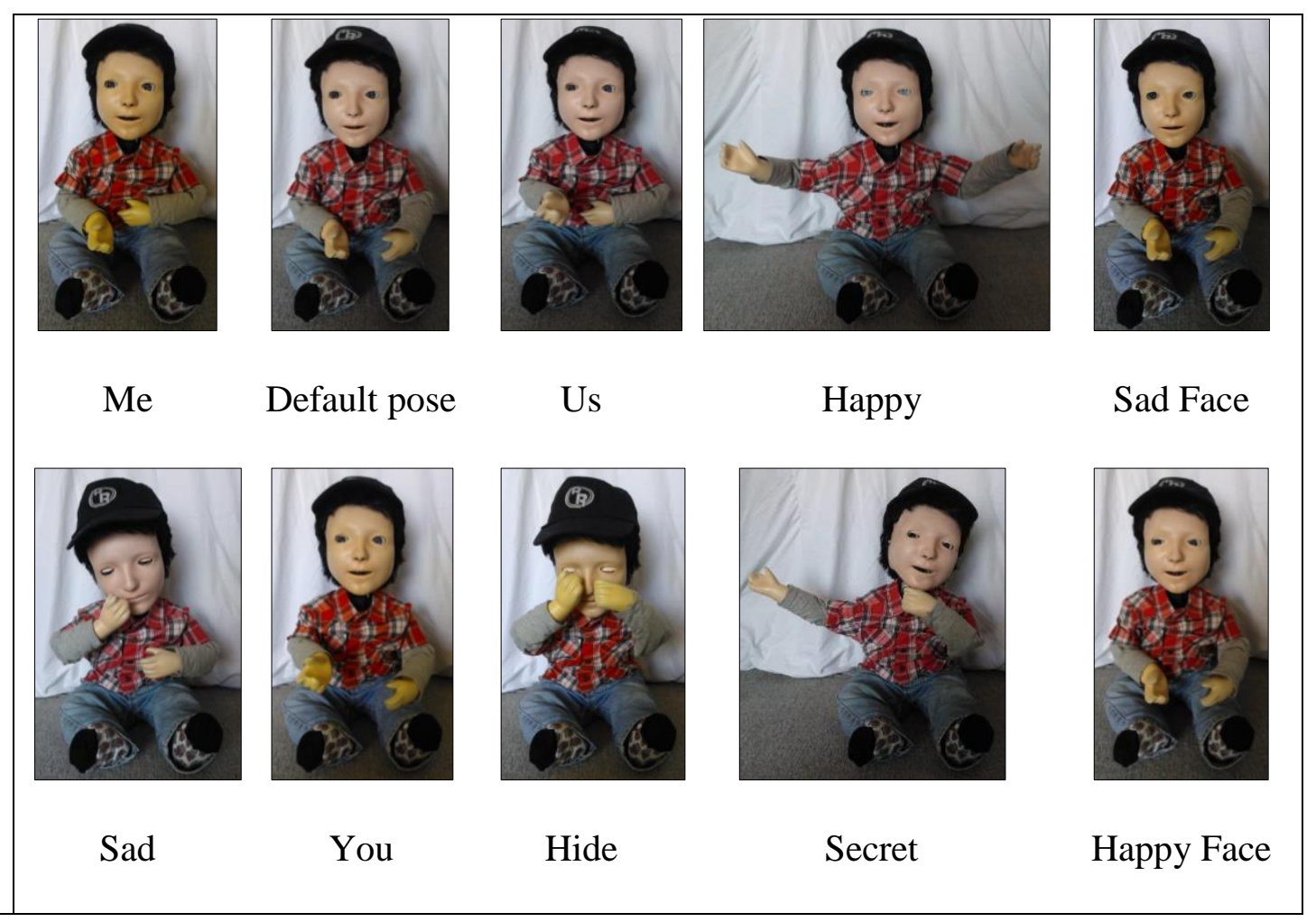

Figure 2. A selection of the robots gestures

\section{Participants}

This study included one adult and ten child participants.

The adult participant: The professional recruited to conduct the field trial was a senior educational psychologist with more than 20 years of experience working for Hertfordshire local authority, over 10 years of which a senior post had been held. The role of a senior educational psychologist is diverse with tasks such as:

- Assessing learning and emotional needs

- Developing and supporting therapeutic and behaviour management programmes 
- Writing statements for children

- Investigative work for tribunals

At a senior level this is extended to training and supervising trainee educational psychologists, and standing in for team leader. The educational psychologist recruited was responsible for supporting students at several mainstream primary and secondary schools, which he attended on a weekly basis, conducting statutory work. At the time of this study the educational psychologist did not support any schools with special previsions, but did have experience of this in the past. The educational psychologist's substantial experience of working with and interviewing children (on a daily basis) made him an ideal candidate to test and provide feedback on a Robot-Mediated Interviewing system.

The child participants: The child participants were ten neurotypical children between the ages of 7 and 8 (6 male and 4 female) that attended a local mainstream primary school in Hertfordshire in the UK where the study was conducted. This age group of children was chosen on the basis of our previous research.

Ethics: Prior to conducting the study informed consent was obtained in writing from all parents of the children participating in the study and the adult interviewer, agreeing to take part in the study. In addition to this, written confirmation was also obtained from the Head of the school to confirm that she was happy for the study to take place in the school and acknowledged that she had seen and was satisfied with the 3 minute video clip that the children were to be shown and questioned about. The ethics protocol number for this study is $\mathrm{COM} / \mathrm{PG} / \mathrm{UH} / 00013$. 


\section{Procedure}

The study was conducted in 3 phases for both the children and the adult interviewer, utilising a pre-trial introduction, trial run, and post-trial debrief.

Pre-trial introduction (the children) - The children were briefly introduced to Kaspar approximately 3 weeks before the trial commenced. This introduction served to familiarise the children with the robot and was also used to explain that Kaspar would be coming in to talk to some of them. This familiarisation phase follows some of the suggestions outlined in (Kyriakidou, 2016). In addition to the Kaspar robot, the children were also given the opportunity to play with some other robotic toys because the children's school term was going to be themed on robots, therefore the class teacher considered that this would be a positive start to the term for the children.

Pre-trial introduction (the interviewer) - The professional interviewer was given a pretrial introduction to explain the procedure for the trial and some training with operating the Kaspar robot. It was explained that all of the children had been briefly introduced to Kaspar as a group to familiarise them with the robot, and that the children were aware that Kaspar would be coming back to talk to them. The interviewer was advised that the children would be shown a 3 minute video clip about which Kaspar would then ask them questions. The interviewer was given a minimal description of the video to help with potential lines of questioning, which was as follows:

"The cartoon depicts two animal characters sitting down in a house with a radio playing in the background. All is quiet and normal before some creatures come into the house and 
cause trouble by eating various items. The two characters eventually combine their efforts to get rid of the creatures that are causing the trouble."

The interviewer was specifically not shown the video prior to the interviews because we wanted to create a situation similar to a real-life interview concerning an event that the child had witnessed, but the interviewer did not. It was explained that once all of the interviews had taken place, the interviewer would get to see the video and would then be able to establish how much information he had managed to elicit from the children. In this pre-trial introduction it was also stated that questioning must only focus on the contents of the video that the children had seen as this was in accordance with our ethics clearance. Following the procedural explanation and demonstration of the system, an interview took place with the educational psychologist to get a detailed description of his professional background and experience. The pre-trial interview questions were designed to establish: what the role involved, how much experience he had in the role, what the most important aspects for the role were, how technology currently plays a part in that role and what expectations he would have of a Robot-Mediating Interviewing system.

The trial (the interviewer and children) - The trial took place 3 days after the pre-trial interviewer introduction. The interviewer was given a brief reminder of the procedure and a short refreshed test of the robotic setup prior to commencing the interviews with the children. The children were taken to and from their class by a teaching assistant. The children were brought out of class to a seating area where the principal investigator was sitting with a laptop. It was explained to the children that they were going to watch a short 
cartoon, and then they would talk to Kaspar, who would ask them questions about the cartoon they had just seen. It was emphasised to the children that it was "not a test" and it was "a bit of fun", as we did not want the children to be stressed. Following this, the children watched the video and then went into another room where they would sit and talk to Kaspar. While the children were talking to Kaspar, the teaching assistant remained in the room but was as unresponsive as possible. The principal investigator was not in the interview room with the children. Each interview lasted between 5 and 10 minutes. The interviews with the children were not recorded because the purpose of this study was to gather feedback from the professional interviewer on their experience and views of the system after the trial.

Equipment installation - Three rooms were used to facilitate this study as can be seen in Figures 3-4. As in previous studies we have conducted a remote connection to the robot and a camera was used to monitor the situation remotely. 

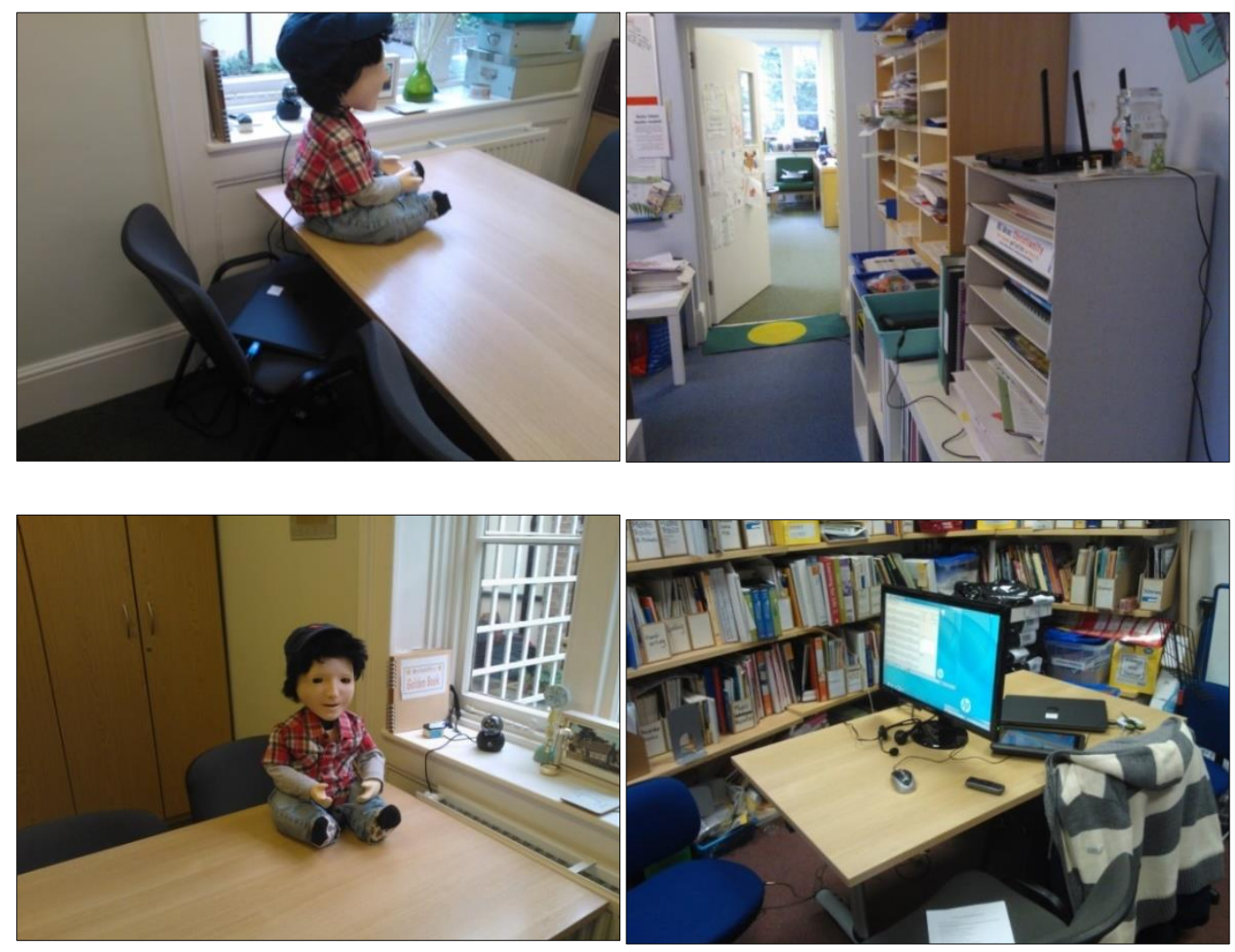

Figure 3. Pictures of study setup 


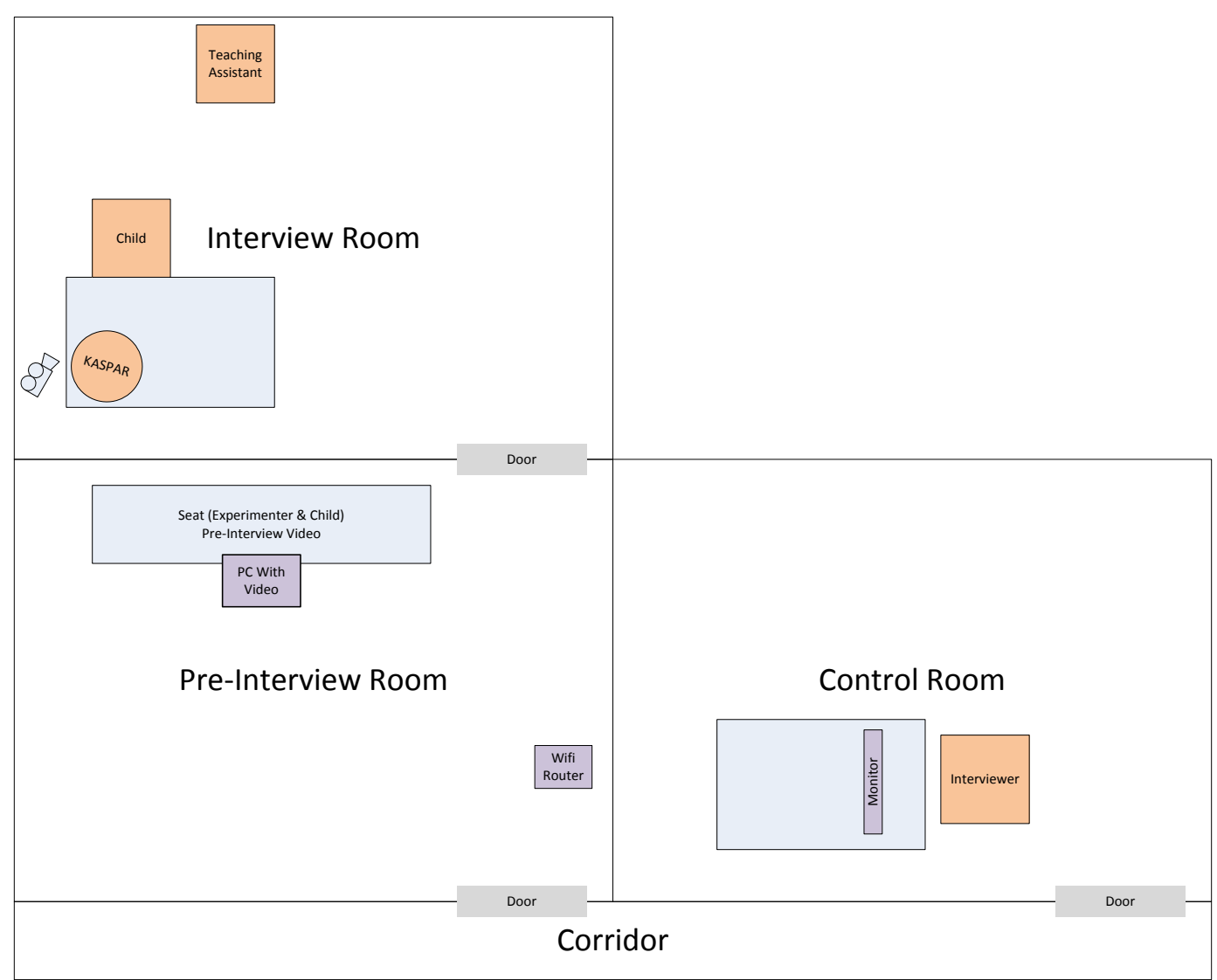

Figure 4. An overview of the room layout and interview setup

Post-trial interview (the interviewer) - After all of the interviews were completed a posttrial interview took place with the educational psychologist to gather feedback on their experience with the system. The purpose of this interview was to establish what they liked about the system, what they did not like about the system, how they felt about this method of interviewing and whether they felt that this approach could be useful in a real-world setting. 
Post-trial debrief (the children) - Two weeks after the interviews had taken place, the children were given a group debrief to show them how the robot worked and were also given the opportunity to play with and control the robot along with a number of other robotic toys. The debrief served as a thank you to the children and the school for taking part in the study, and was also educational in teaching the children a little bit about robots.

\author{
Measures \\ The data collection for this study is based on feedback from the expert interviewer. The pre \\ and post-interviews with the educational psychologist were recorded and later transcribed \\ for analysis. When analysing the transcriptions the key points were logged and are \\ discussed in the results section of this paper.
}


Results

$\underline{\text { Interviewer background (pre-trial interview) }}$

This section is broken down by the aspects of what we asked the educational psychologist.

\section{What are the most difficult aspects of being an educational psychologist: The}

educational psychologist explained that writing reports on the children was the most time consuming aspect of the role, but the real challenge is ensuring that the child's needs are met in terms of behaviour problems, learning difficulties, or social emotional problems. The most difficult cases are tribunal cases where there is a conflict between what the parents want and what the authorities are offering. The educational psychologist explained that information is obtained from a number of sources including teachers, parents and the child, to create a complete picture of the child. The educational psychologist considered that the most important part of an investigation is the individual work with the child, because it is important to understand their perspective rather than relying on other people's perceptions of the child.

Who do educational psychologists work with: Depending on the case and the needs of the child, educational psychologists often work with people in other professions such as:

- Speech and language therapists

- Occupational and physiotherapists

- Paediatricians

- Social workers 
- Very occasionally police officers

The experience of working with professionals from a variety of disciplines is particularly beneficial for the purpose of this research, because he is likely to have a knowledgeable view of how a Robot-Mediated Interviewing system might be useful to other professionals.

\author{
What children are the most difficult to deal with: The educational psychologist \\ explained that secondary school children around the age of 13 and 14 who are showing \\ very disturbed behaviour can be the most challenging whereas younger children are often \\ much easier to work with. Severe autism can sometimes present a challenge, particularly \\ when trying to establish the triggers for disruptive behaviour. Typically developing \\ children will often be able to say what is bothering them, whereas children with severe \\ autism often cannot talk and sometimes will not even relate to you, making them very \\ difficult to communicate with.
}

What factors affect communication: Sometimes children are very oppositional because they see the educational psychologist as an authority figure. The educational psychologist stated that it sometimes helps to tell the child "I don't belong to the school, so you can tell me anything you want", particularly if the child is not getting on with their class teacher very well and is worried about getting reprimanded. Children can be very challenging and difficult to communicate with because of unknown circumstances at home such as: separation of parents, a new baby is born in the family and the child is jealous, or dealing with loss or bereavement due to the death of a parent or sibling. Two particular aspects that the educational psychologist believed that a robot-mediated interviewing system such as 
Kaspar could assist with are connecting with the child and not being seen as an authority figure. Because a robot such as Kaspar will be smaller than the child and would be considered closer to the child's age in terms of appearance, this may mean that the child will both view the robot as more of an equal to them, thus helping to connect with them and eliminating the potential of being viewed as an authority figure. Another aspect affecting communication is that children will sometimes view the educational psychologist as stranger. One approach to solve this problem is for the educational psychologist to be seen by the child talking to the teacher, showing the child that the he is accepted by the class teacher and is fairly familiar. Because the teacher is a trusted person, if the educational psychologist appears to be trusted by the trusted person, then the child will see that it is ok for them to talk to him.

\section{What are your expectations of a Robot-Mediated Interviewing system: The}

educational psychologist believed that the novelty effect would be "tremendous" and that the children were likely to be very excited, although some children may be scared if they were very young. It was explained that all of the children had been briefly introduced to Kaspar as a group, so that they would be familiar with Kaspar. The educational psychologist believed that the children he would be working with, between the ages of 7 and 9, would be comfortable with the robot. The educational psychologist also believed that the lack of response in the robot's face could be quite inhibiting for some children, because sometimes children will be looking for positive non-verbal feedback, and if they are not getting it they may get worried and switch off or withdraw. The educational psychologist also believed that some of the children might want to play with or even attack 
the robot to see what happens. Overall the educational psychologist thought that children between 7 and 9 years of age would probably be fascinated by the robot because they have already seen it before and that it would be good fun for them.

\author{
What preconceptions in terms of the advantages and limitations do you have about \\ the Robot-Mediated Interviewing approach: The system would probably be useful for \\ children with autism who find it really difficult to make eye contact and understand facial \\ expressions, as they may be able to look at it much more easily. In addition to this, the \\ novelty effect to children with autism might be particularly useful as some children with \\ autism switch off to human voices, but may not with a robot. The educational psychologist \\ believed that in the case of typically developing children the robot might take the place of a \\ trusted doll. Children sometimes talk to their dolls and their teddy bears and tell them \\ things that they would not tell an adult. However, in this type of situation it would be \\ unlikely to happen immediately as the first experience would most likely be about fun.
}

\title{
System feedback (post-trial interview)
}

Immediately after the interviews with the ten children were completed, a post-trial interview was held with the educational psychologist to gather feedback on his experience, views and thoughts on the interviews he had conducted. This feedback has been broken down into categories.

How did the educational psychologist find the interviews: The educational psychologist described the interviews as "fascinating", because all the children were very different in 
terms of memory, understanding and vocabulary. Some of the children had an excellent vocabulary and it was very clear that they understood words such as "termites"; in contrast, other children seemed to have forgotten large parts of the video, and did not know the name of the insects. One child referred to them as "flies" whilst another referred to them as "mice". The educational psychologist found it fascinating to see how patchy short term memory was for the children, mentioning that it was quite difficult to draw information from some of them, possibly because of personality characteristics.

\section{How much information did the educational psychologist manage to acquire from the}

children: The educational psychologist managed to acquire much less information than expected, but believed that this may have been due to the novelty effect of the robot and nature of the activity. Although the children had been introduced to the robot prior to the study, this may not have been enough, and the novelty effect may have interfered with some of the children's memory. Despite the lack of information elicited from the children, our three previous studies found that there were no significant differences in the information the children provided a robot (Wood, Dautenhahn, et al., 2013a, 2013b; Wood, Dautenhahn, Rainer, et al., 2013). With regards to the details that the children recalled about the video, all of the children stated that the cartoon was a Tom and Jerry cartoon. One of the children was particularly good at recalling details of the video but still missed some major points which would have been very clear and visual in the video. The educational psychologist noted that even when prompting the children with information received in prior interviews in an effort to get them to say more, some of the children still said that they could not remember. However this can be viewed as positive because if the 
children could genuinely not remember and could not be coerced by the robot, this could indicate that children are less likely to be led by a robot, an argument that is supported by Bethel et al. who found that people were less likely to be misled by robotic interviewers than human interviewers (Cindy L Bethel et al., 2013). The educational psychologist believed that sufficient pre-interview exposure to the robot would be needed if it was to be used as an interview tool in order to get the children acclimatised to the robot.

How did the children react to the robot: There were mixed reactions to the robot with some children appearing to be slightly wary of the robot, whist other children really enjoyed the interaction with the robot. The educational psychologist stated that some of the children were quite willing to talk, but their inability to remember details about the video and answer the questions seemed to put them off. One child in particular was interested and started talking about himself and the things in his head. The educational psychologist explained that although the children were told that the activity was not a test, some children will still feel uncomfortable about not having the answer to the question they are being asked. Children of this age are particularly sensitive to this situation. The educational psychologist went on to say that different children have different ways of dealing with being unable to answer a question. He explained that some children will start talking more quietly, while others will start to fabricate answers, which in evidence-gathering situations would obviously present a problem.

Was controlling the robot as expected: The educational psychologist stated that the system provided an "amazingly interesting way of remotely looking at the children from a 
different angle" and explained that the system took some time getting used to, but it got easier the more that he used the system, however there was a slight time delay in the system. The educational psychologist suggested that it would be better to be able to see the robot as well as the child, because it was sometimes difficult to know what position the robot was in. This would also aid in assisting the co-ordination of the robot's gestures during the interaction. The educational psychologist stated that there was occasionally some lag time between the robot's gesture and the feed from the camera that was focused on the children. The educational psychologist explained that eliminating the lag time coupled with having an additional camera feed focused on the robot would make the system a lot easier to use and help to ensure the gestures were well coordinated. The educational psychologist also stated that because he was focused on interviewing the child, there were a few occasions when he realised that he had not made the robot do anything for some time; having a constant view of the robot's state would help to elevate this problem. Exploring the possibilities of enhancing the system in the future, we asked the educational psychologist how he would feel about a system that was literally attached to the interviewer, with sensors that could detect arm and potentially head movements. The educational psychologist believed that this would be an excellent idea, and would make the interaction with the system from the interviewer's perspective much more natural. To establish the most positive aspects of the system, we asked the educational psychologist what he liked about this method of interviewing. The educational psychologist stated that he liked this method of interviewing because it was fun. The educational psychologist explained that he did not have any major expectations of the system and had expected more of the children not to take the robot seriously. He believed that 8 out of the 10 
children did take the robot seriously. The educational psychologist stated that despite the interviews being a slightly odd, though fun situation, the children were focused on what they were being asked and actually responded as if it was an adult asking them questions, rather than going off at a tangent. The educational psychologist found this interesting, particularly because it was very clear that the robot was a toy. The educational psychologist explained that the system gave him the opportunity to really focus on facial expressions of the children. When talking to someone in an interview, the interviewer cannot just stare at the person as this would make them uncomfortable. When looking via a camera from a third person perspective, it allows the interviewer to totally focus on the child's face and non-verbal feedback.

\section{What would be the prime age group for this type of approach of interviewing: The} educational psychologist believed that a robot-mediated interviewing approach could be suitable for children between the ages of 5 and 7, but emphasised the importance of familiarising young children with the robot first in order to make them feel safe. However he noted that the information younger children provide may be less reliable, but it would depend on the kind of interview. Further to this he was quite adamant that a RobotMediated Interviewing approach would not be suitable for children over the age of 9.

\section{What the educational psychologist did not like about this method of interviewing: The} educational psychologist stated that he enjoyed using the system although there were some technical difficulties with the sound and initially he could not hear the children clearly. He reiterated that being able to see Kaspar on the screen as well as the child would make the 
system much better, but there was not anything he particularly disliked about the system. We asked the educational psychologist, "What would you change about the system?". In addition to this he stated that the sound issues would need to be resolved and that there were some lagging issues with regards to the video streaming of the child and the movements of the robot, but other than this the system was fine.

\section{Did the educational psychologist think this approach could be used in a real-world}

setting: The educational psychologist believed that the system could be considered for real-world applications provided the inadequacies of the system were rectified first. The educational psychologist also confirmed that he felt the system might be useful for child protection interviews and acquiring disclosures. He explained that the system might be useful for simply getting children to talk about their worries, not necessarily for child protection issues. He stated that the children might feel able to tell the robot about things that they are worried about, adding that some children have huge anxieties about different matters in their lives, and sometimes the children are frightened to talk about these concerns. Quite often once the child has talked about them it becomes apparent that these anxieties are about quite minor things, but once the child has expressed these concerns it is something that they can be helped with. The educational psychologist believed that a robot-mediated interviewing system might be useful for highly anxious children, just to have a chat, and that the system would probably be suitable for children who are reluctant to talk, such as children with autism and possibly selective mutes. He explained that selective mutes will not talk in particular settings. For example, the child may talk perfectly normally at home but will not talk at school. It is extremely difficult to get 
selective mutes to talk; eventually the child might whisper to a peer, or whisper to a trusted adult. The educational psychologist believed that it would be worth investigating the possibility of using Kaspar with that group of children, because it may be a useful tool for getting these children to talk. He stated that the system provided an interesting and totally different way of looking at children's memory and explained that there are learning and memory tests that educational psychologists conduct with children, but often these tests are not very interesting for the children. By using a robot such as Kaspar this might be a good way of testing children's memory, and making the test enjoyable and interesting for the children.

\section{Would a tool like this be useful for professionals interviewing children: The}

educational psychologist stated that he could envisage some uses for the system in his profession, explaining that finger puppets and toys are sometimes used to try and help children to talk and communicate, and that using a robot would be a step on from this. With regards to the professions where the system may be applicable, the educational psychologist believed that the system could potentially be useful for educational psychologists, police, social services, education, and potentially speech and language therapists. 


\section{Discussion}

\section{Summary of findings}

This field trial with an educational psychologist found that similarly to previous studies, most of the children responded to the robot and treated the activity seriously. The educational psychologist was surprised by how little information many of the children could recall about the video that they had seen, however the educational psychologist suspects that this was not due to the robotic interviewer, but rather it was the nature of the activity itself. The educational psychologist believed that the system would benefit from further development to resolve some of the technical difficulties such as sound and lagging issues, but could see that the system had real-world potential if it was developed further. One of the potential advantages outlined by the educational psychologist that had not previously been considered was the ability for the interviewer to focus on the children's non-verbal feedback from a third person perspective.

When talking to someone in an interview the interviewer cannot just stare at them as this would make them uncomfortable. Using a camera with a remote connection and speaking via the robot enables the interviewer to focus much more on the body language of the child. The educational psychologist believed that potential applications that this system would be useful for included child interviews with the police, social services and educational psychologists, noting that the system would probably be best suited to children with conditions that create communication difficulties such as autism. The educational psychologist also believed that the system may have wider applications outside of a child protection context and could be useful for children who are selective mutes and children 
that suffer with anxiety who find it difficult to talk to adults. An additional application area where the robot could be used that the educational psychologist outlined was for testing children's memory, because using a robot would be a more interesting and engaging way to conduct these types of tests with children. Overall the feedback from the educational psychologist indicates that robot-mediated interviews are a possibility and may have potential for real-world applications. Additionally the educational psychologist believed that the system may also have a wider range of applications outside of child protection and encouraged us to explore these possibilities further.

Studies to date on Robot-Mediated Interviewing systems have primarily focused on researchers conducting studies with participants directly with their systems (Cindy L Bethel et al., 2013; C. L. Bethel et al., 2011; Wood, Dautenhahn, et al., 2013a, 2013b; Wood, Dautenhahn, Rainer, et al., 2013). The research conducted in this study takes the use of Robot-Mediated Interviews a step further by putting the system into the hands of a potential real-world user and gathering feedback about the usability of the system and most likely applications. Gathering real-world opinions from a professional who would potentially use the system in the real-world is an important step in ascertaining if a RobotMediated Interviewing system has practical applications and what would be required from the system from a usability perspective to make the transition from research to a real-world system, a viewpoint that is reflected by (Conti et al., 2017).

\section{Conclusion}

The primary research questions that this study aimed to answer were: 
RQ-1) What would experts working with children require from a Robot-Mediated Interviewing system?

RQ-2) Can robots such as Kaspar be used in an interview setting by an expert working with children?

To answer our first research question the educational psychologist testing the system in this study reiterated the points stated in user panels in our previous study (Wood et al., 2014, 2016) and also stated that the system would benefit from having a camera to monitor the status of the robot. In addition to this the educational psychologist outlined additional areas where the system may be of use which were for children that are selective mutes and for educational psychologists conducting memory tests because a robot may be a more interesting way to conduct the test with the children. Ultimately this study has shown that robots can be used by professionals to interview children answering our second research question.

\section{Limitations}

Although this study with an educational psychologist has provided us with valuable feedback, it would have been desirable to test the system with multiple professionals from a variety of backgrounds in order to obtain views from different perspectives. The impact of different settings and contexts for the interviews could also be investigated, as well the use of robot-mediated interviews by professionals in their daily work with children. Last but not least, future work would also benefit from the opportunity to conduct multiple cycles of feedback, redesign, implementation and testing to further improve the system. 


\section{Acknowledgements}

We would like to thank the staff and children of the school who participated in this study along with the educational psychologist who conducted the field trial with the robot. 


\section{References}

Screaming Bee. MorphVOX Pro. Retrieved from http://www.screamingbee.com/product/MorphVOX.aspx

Bethel, C. L., Eakin, D. K., Anreddy, S., Stuart, J. K., \& Carruth, D. (2013). Eyewitnesses are misled by human but not robot interviewers. Paper presented at the Proceedings of the 8th ACM/IEEE international conference on Human-robot interaction.

Bethel, C. L., Henkel, Z., Stives, K., May, D. C., Eakin, D. K., Pilkinton, M., . . StubbsRichardson, M. (2016). Using Robots to Interview Children about Bullying: Lessons Learned from an Exploratory Study. Paper presented at the Presentation in the Special Session on Child-Robot Interaction at the 25th IEEE International Symposium on Robot and Human Interactive Communication (RO-MAN 2016).

Bethel, C. L., Stevenson, M. R., \& Scassellati, B. (2011). Sharing a Secret: Interactions Between a Child, Robot, and Adult. Paper presented at the Presented at the Children with Robots Workshop at the 6th ACM/IEEE International Conference on HumanRobot Interaction, Lausanne, Switzerland.

Cabibihan, J.-J., Javed, H., Ang Jr, M., \& Aljunied, S. M. (2013). Why robots? A survey on the roles and benefits of social robots in the therapy of children with autism. International Journal of Social Robotics, 5(4), 593-618.

Chevalier, P., Martin, J.-C., Isableu, B., Bazile, C., \& Tapus, A. (2017). Impact of sensory preferences of individuals with autism on the recognition of emotions expressed by two robots, an avatar, and a human. Autonomous Robots, 41(3), 613-635.

Conti, D., Di Nuovo, S., Buono, S., \& Di Nuovo, A. (2017). Robots in education and care of children with developmental disabilities: a study on acceptance by experienced and future professionals. International Journal of Social Robotics, 9(1), 51-62.

Costa, S., Lehmann, H., Dautenhahn, K., Robins, B., \& Soares, F. (2015). Using a humanoid robot to elicit body awareness and appropriate physical interaction in children with autism. International Journal of Social Robotics, 7(2), 265-278.

Costescu, C. A., Vanderborght, B., \& David, D. O. (2014). The effects of robot-enhanced psychotherapy: A meta-analysis. Review of General Psychology, 18(2), 127.

Esteban, P. G., Baxter, P., Belpaeme, T., Billing, E., Cai, H., Cao, H.-L., . . . De Beir, A. (2017). How to build a supervised autonomous system for robot-enhanced therapy for children with autism spectrum disorder. Paladyn, Journal of Behavioral Robotics, 8(1), 18-38.

UK Government. (2011). Achieving Best Evidence in Criminal Proceedings: Guidance on Interviewing Victims and Witnesses, and Using Special Measures. Home Office Criminal Justice System

Hsu, C.-W., \& Teoh, Y.-S. (2017). Investigating Event Memory in Children with Autism Spectrum Disorder: Effects of a Computer-Mediated Interview. Journal of autism and developmental disorders, 47(2), 359-372.

Jones, L., Bellis, M. A., Wood, S., Hughes, K., McCoy, E., Eckley, L., . . Officer, A. (2012). Prevalence and risk of violence against children with disabilities: a systematic review and meta-analysis of observational studies. The Lancet. 
Kyriakidou, M. (2016). Discussing robot crime interviewers for children's forensic testimonies: a relatively new field for investigation. AI \& SOCIETY, 31(1), 121126.

Martínez-Miranda, J., Pérez-Espinosa, H., Espinosa-Curiel, I., Avila-George, H., \& Rodríguez-Jacobo, J. (2018). Age-based differences in preferences and affective reactions towards a robot's personality during interaction. Computers in Human Behavior, 84, 245-257.

Mead, R., Grollman, D. H., Lim, A., Yeung, C., Stout, A., \& Knox, W. B. (2018). HRI 2018 Workshop: Social Robots in the Wild. Paper presented at the Companion of the 2018 ACM/IEEE International Conference on Human-Robot Interaction.

Meghdari, A., Alemi, M., Khamooshi, M., Amoozandeh, A., Shariati, A., \& Mozafari, B. (2016). Conceptual design of a social robot for pediatric hospitals. Paper presented at the Robotics and Mechatronics (ICROM), 2016 4th International Conference on.

Mencap. (1999). Submission to the Sex Offences Review.

Pennisi, P., Tonacci, A., Tartarisco, G., Billeci, L., Ruta, L., Gangemi, S., \& Pioggia, G. (2015). Autism and social robotics: A systematic review. Autism Research.

Robins, B., \& Dautenhahn, K. (2014). Tactile interactions with a humanoid robot: novel play scenario implementations with children with autism. International Journal of Social Robotics, 6(3), 397-415.

Robins, B., Dautenhahn, K., \& Dickerson, P. (2009, February 1-7). From isolation to communication: A case study evaluation of robot assisted play for children with autism with a minimally expressive humanoid robot. Paper presented at the Proc. the Second International Conferences on Advances in Computer-Human Interactions, ACHI 09, Cancun, Mexico.

Sartorato, F., Przybylowski, L., \& Sarko, D. K. (2017). Improving therapeutic outcomes in autism spectrum disorders: Enhancing social communication and sensory processing through the use of interactive robots. Journal of psychiatric research, 90, 1-11.

Wood, L. J., Dautenhahn, K., Lehmann, H., Robins, B., Rainer, A., \& Syrdal, D. S. (2013a). Robot-Mediated Interviews: Do robots possess advantages over human interviewers when talking to children with special needs? Paper presented at the International Conference on Social Robotics, Bristol, UK.

Wood, L. J., Dautenhahn, K., Lehmann, H., Robins, B., Rainer, A., \& Syrdal, D. S. (2013b). Robot-Mediated Interviews: Does a robotic interviewer impact question difficulty and information recovery? Paper presented at the 12th European AAATE Conference, Vilamoura, Portugal.

Wood, L. J., Dautenhahn, K., Rainer, A., Robins, B., Lehmann, H., \& Syrdal, D. S. (2013). Robot-Mediated Interviews-How Effective Is a Humanoid Robot as a Tool for Interviewing Young Children? PLOS ONE, 8(3), e59448.

Wood, L. J., Lehmann, H., Dautenhahn, K., Robins, B., Rainer, A., \& Syrdal, D. S. (2014). Robot-Mediated Interviews with Children: What do potential users think? Paper presented at the AISB Symposium New Frontiers in HRI.

Wood, L. J., Lehmann, H., Dautenhahn, K., Robins, B., Rainer, A., \& Syrdal, D. S. (2016). Robot-Mediated Interviews with Children: What do potential users think? Interaction Studies, Vol. 17:3, pp. 439-461. 
